\title{
Mars System Science Why Mars Remains a Compelling Target for Solar System Science
}

A White Paper prepared for the 2023-2032 Planetary Science and Astrobiology Decadal Survey on behalf of the Mars Exploration Program Analysis Group (MEPAG)

by the MEPAG Steering Committee.
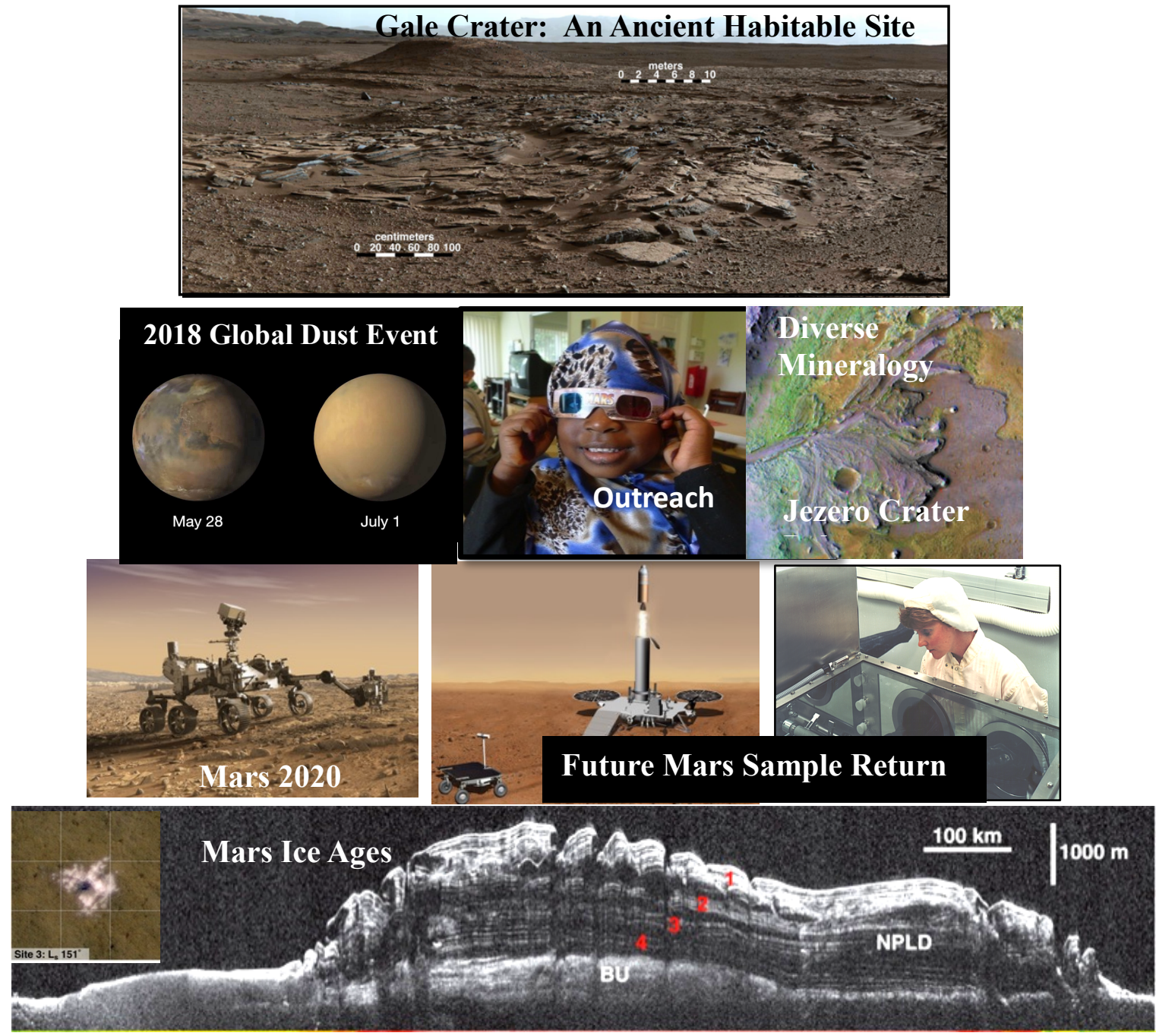

Contact information: Dr. R. Aileen Yingst, MEPAG chair, Planetary Science Institute; 920-360-3627; yingst@psi.edu

15 July 2020

Citation: MEPAG (2020), Mars System Science: Why Mars Remains a Compelling Target for Solar System Science, R.A. Yingst, ed., 7 p. white paper posted July, 2020 by the Mars Exploration Program Analysis Group (MEPAG) at https://mepag.jpl.nasa.gov/reports.cfm?expand=decadal. 


\section{Executive Summary}

Mars is and remains a compelling target for planetary exploration because it can effectively address many of our most fundamental questions in solar system science. Mars is an excellent and readily accessible candidate for searching for evidence of past or present extraterrestrial life; a unique place to explore how climates and atmospheres form and change through time; a key data point for exploring the formation and evolution of terrestrial planets; and a laboratory for characterizing the variables that feed into defining solar system habitable zones. Uniquely, Mars retains, almost in its entirety, a record of planetary evolution not found in such pristine form elsewhere in the solar system (including Earth), and one that is unavailable for study outside the solar system - the record of a planet that once hosted habitable surface environments similar to Earth's, but, through a confluence of events, evolved environmental conditions of less certain habitability. Mars might be seen as the Earth that almost was, and understanding why this was so is crucial to understanding life's origins by deciphering what is required for life to evolve and thrive. All environments on early Earth also existed on early Mars, making Mars the most accessible extraterrestrial planet where habitability coexisted with the potential for life to arise.

Because the record of its geologic and climatic history is retained, and because there is current activity (occurring within context) whose systematic observation will illuminate the processes of change operating today and in the past, Mars can challenge theory with data in ways that will result in a better understanding of all terrestrial planets, but especially of Earth.

\section{Prologue}

In the last few decades, planetary science has begun the inexorable progression from examining the solar system as a discrete collection of bodies, to approaching our local neighborhood as one example of solar system formation and evolution. The science questions we ask are broadening to more fundamental ones that recognize the complex, interconnected and interdisciplinary processes involved in the formation and evolution of planetary bodies. This heralds the deparochialization of planetary science into a systems approach with a more universal perspective, both figuratively and literally.

While every planet brings unique and important information to bear when answering such fundamental overarching science questions, only Mars preserves a readily accessible record of that early history where climate evolution was most similar to early Earth, while also addressing a wide range of comparative planetology questions. In the 1980 s science reached a turning point when Earth System Science was realized. That turning point for Mars System Science is now; we are just beginning to detail the drivers of Mars' evolution.

We first discuss the current state of Mars science in the context of the highly successful Mars Exploration Program. We then address the way that Mars answers five fundamental science questions about the formation and evolution of the solar system: (1) What pathways can lead to habitable environments, and ultimately life? (2) How do climates and atmospheres form, evolve and change through time? (3) What is the internal structure, origin and evolution of terrestrial planets? (4) How is the solar system representative of planetary systems in general, specifically in terms of habitable zones? and (5) What is needed to design and implement human missions?

\section{State of Mars Science in the Last Decade}

The Mars Exploration Program (MEP) represents a systematic approach to science - a deliberate, strategic program to ensure that high priority science is achieved, that science can be advanced in logical, progressive stages, and that scientists and engineers can plan ahead, not to the 
next three-year proposal, but a decade in advance (Hubbard, 2011). The power and success of the MEP approach has been the ability to lower risk and strengthen science impact by interleaving and overlapping different but complementary mission types, each one providing data to support the science goals of the next (cf. MASWG report), from orbital mapping missions and early rovers to Mars Sample Return, which takes a crucial step this decade with the Perseverance rover (McSween et al., 2020). The MEP can serve as a template for a systematic approach to all other bodies and groups of bodies, as its success over the past decade has expanded our knowledge and focused the science imperative of Mars to tackle new important directions. Major accomplishments in Mars science realized under that program since Visions \& Voyages are listed below:

- Mars was a habitable planet (Is it still?). In this, rover exploration has been game-changing. A habitable environment was recorded in Curiosity's first drilled rock. In situ analysis of drilled samples in Gale crater revealed the necessary elemental building blocks of life $(\mathrm{C}, \mathrm{H}, \mathrm{N}, \mathrm{O}, \mathrm{P}, \mathrm{S})$ in a low salinity, circum-neutral pH water-rich environment (Grotzinger et al., 2014; Rampe et al., 2020). The presence of lakes and sedimentary rocks and long-existing groundwaters was confirmed (Grotzinger et al., 2015; Stack et al., 2019; Edgar et al., 2020), illuminating the relationships between fluvial, deltaic and lacustrine settings. Traces of methane in the atmosphere (Webster et al., 2018) and organic molecules released from sediments (Freissinet et al., 2015; Eigenbrode et al., 2018; Szopa et al., 2020), both of unknown origin, have also been detected.

- Mars was habitable for a very long time. Habitable environments with water activity persisted, perhaps episodically, well into the Amazonian (e.g. Skok et al., 2010; Kite et al., 2017). In the last decade, orbital and in situ observations revealed evidence for active ground water systems that persisted into geologically recent eras and sometimes interacted with the surface (e.g. Ehlmann and Edwards, 2014; Arvidson, 2016; Martin et al., 2017; Ehlmann, 2019). Analyses point to the potential role of ice in a colder but still wet environment, including the action of ground water, into geologically recent eras (e.g., Wilson et al., 2016).

- Mars has the potential to preserve biosignatures. Sedimentary organic matter has been found to be preserved in $>3$ Gyr sedimentary rocks (Eigenbrode et al., 2018), suggesting that some biochemical signatures, if ever present, could be preserved. Though the Martian surface is exposed to ionizing radiation that could destroy sensitive organic matter, the current surface is active in places (surface exposure ages $<\sim 100$ Myr (Farley et al., 2014)) where organic materials might be retained in the near surface, or accessed below scarps.

- Mars' obliquity variations are recorded in the geologic record. For example, internally and externally exposed layering in the polar caps, and of subsurface ice in mid-latitudes, can now be semi-quantitatively linked to variations in Mars obliquity and orbital eccentricity on time scales of $10^{5}$ to $10^{7}$ years (Smith et al., 2016; Becerra et al., 2019; Buhler et al., 2020).

- Escape to space has resulted in massive loss of an early Martian atmosphere. The loss of water is driven both by external solar wind interactions and variable transport into the upper atmosphere from below, particularly as loss is enhanced by orders of magnitude during major dust storms (e.g., Chaffin et al., 2017; Heavens et al., 2019), demonstrating that escape is a key climatic driver.

- The known extent of surface, near-surface, and deep deposits of volatiles continues to expand. At the surface, 1-3 wt \% water is present in Martian fines and dust (Leshin et al., 2013; Ehlmann et al., 2016). Water ice in the subsurface is exposed in new impact craters and in debris-covered ice cliffs (Dundas et al., 2018). Extensive deposits of ice persist at lower latitudes than previously known or predicted from climate models (Bryne et al., 2009; Bramson et al., 2015). The amount 
of $\mathrm{CO}_{2}$ ice trapped in the south polar deposits, if released, would more than double the present atmospheric mass (Phillips et al., 2011; Putzig et al., 2018).

- Dynamic change occurs on Mars today. Observations have the spatial and temporal resolution to reveal block falls, sand movement (e.g. Bridges et al., 2012; Geissler et al., 2012; Baker et al., 2018), seasonal methane releases (Webster et al., 2018), hydration and dehydration of salts (Gough et al., 2020), recurring slope lineae (e.g. McEwen et al., 2014), the roles of $\mathrm{CO}_{2}$ ice volatilization in surface erosion in high latitudes (Diniega et al., 2013; Dundas et al., 2017), the quasi-regular occurrence of regional dust storms, and the episodic occurrence of planet-wide dust events, the latest in 2018. These are modern processes that have produced change throughout Mars history.

\section{Mars as a Compelling Target for Overarching Planetary Science Questions}

\section{Mars is an ideal proving ground to address (and answer?) what pathways can lead to habitable environments, and ultimately life.}

How has the availability of habitable/inhabited environments on and within planets changed with time? Mars provides a unique and crucial data point as a world that was once more habitable than now, because it retains much of its geologic record, allowing us to concretely determine the driving processes that enhanced or limited biological potential. We can also explore the edges of habitability, including searching for the signs of present and past life.

Habitability. Mars and Earth represent two planets that had habitable conditions simultaneously in one solar system, a possibility with profound implications for life in the universe. The earliest undisputed evidence for life on Earth dates $\sim 3.7 \mathrm{Ga}$, although possible biotic remains have been dated as early as prior to $4 \mathrm{Ga}$. However, the rock record on Earth prior to $\sim 3.5 \mathrm{Ga}$ has been largely destroyed or altered through plate tectonics, metamorphism, or other processes, and what fragments remain appear without original geologic context. In contrast, Mars records the evolution of potentially habitable environments in the solar system's first two billion years in a pristine, relatively accessible form (Tanaka et al., 2014). Mars therefore has a unique archive of ancient evidence, in lithologic and geologic context, of water/rock interaction, sedimentation, and other processes crucial for forming habitable environments, as well as a record of the evolution of climate and recognizable habitats such as river deltas, lakes, and hydrothermal deposits. To answer the broad questions of which conditions create a habitable environment, how habitable worlds evolve over time, if and when they become inhabited, and how and why habitats shrink, we must understand which geophysical and climatic conditions were the deciding factors in habitability. Mars is an ideal proving ground for hypotheses regarding what variables are important in developing and sustaining a habitable environment (e.g. Ehlmann et al., 2016).

Signs of Life. Does extraterrestrial life exist, and has it existed in the solar system? If life has developed elsewhere, where and when was it, and why, and what are/were its characteristics, does/did it have Earth-like biochemistry, and how do we detect and confirm its presence? If life is not found elsewhere, why not? As we have gained experience and knowledge, a lively debate has developed in the astrobiology community regarding the best method for approaching the "life" question: do we search for extinct life, extant life, or the organics that might generate, sustain, and signal life (Davila et al., 2020)? Mars is a crucial test case for the fundamental question of what is required for life to arise, as it remains the only place within short-term exploratory reach where we might conduct all three of these searches and expect answers.

Extinct life. Mars holds a distinctive position as the only planet besides Earth where habitability coexisted with the potential for life to arise in multiple, distinct environments 
throughout space and time, including every environment that existed on early Earth. Recent missions to Mars have demonstrated that there are many regions that may once have been habitable; Spirit (e.g. Ruff et al., 2020) and Opportunity data (e.g. Arvidson et al., 2016) have suggested, and Curiosity data confirmed (e.g. Grotzinger et al., 2014), at least one; there are many more suggested by observations from orbit (Amador \& Ehlmann, 2020 and references therein). Our current and growing body of knowledge regarding these potential regions of past habitability is key to building up the contextual knowledge required to confirm a positive, or having confidence in a negative, result for ancient Martian life.

Extant life. The four Viking biological experiments represented the first purposeful in situ tests for extraterrestrial life; the general consensus is that the results of these tests could be explained by abiotic chemical processes, but also that results were inconclusive. The Martian subsurface may even now be on the edge of habitability for life (e.g., Cockell, 2014). Due to its obliquity cycles, Mars had twice the atmospheric pressure it now does within a geologically recent period of time (500,000 years ago), bringing more of the surface above the triple point of water (e.g., Buhler et al., 2020). Should life have arisen in previously habitable regions, some sites might remain habitable during periods of high obliquity, e.g., mid-latitude ground ice, the poles (Stoker et al., 2010). Because even roving missions to Mars are highly localized on a planetary scale, most of the surface (and essentially all of the subsurface) remains unexplored with respect to extant life.

Organics. Finally, Mars is ideally suited to yield fundamental information regarding organic chemical evolution. The distinction between extant and extinct life emphasizes the metabolic state of microbes, an assumption that may force requirements on any search for evidence of life that might not be necessary. Instead, to a first degree the issue might be distilled down to whether Martian microbes have ever been present. Organic chemistry analysis can detect very tiny amounts of key materials, making it more sensitive than most other tests for evidence of life. Additionally, understanding abiotic organic chemical evolution and genesis (e.g., Steele et al., 2018) may be as important to answering the life question as the actual search for life. For example, the discovery of complex, abiotic organic chemistry would add to a growing body of evidence that the biochemical building blocks of terrestrial life might be universally available.

In short, Mars is an excellent candidate for searching for evidence of extraterrestrial life. Current data show Mars once had both the building blocks of life and habitable regions, and may still have them, perhaps in the ice, in caves, or deeper underground. Mars is the one place in the solar system where we can look for extant life in depth and at depth. We have literally only scratched the surface in understanding the habitability and life potential of the body most analogous to Earth.

\section{Mars is a unique place to explore how climates and atmospheres form, evolve and change through time.}

Ancient Climate. How do atmospheres develop, and what drives their composition and dynamics? What are the fundamental physical processes and rates of surface-atmosphere exchange of material and volatiles on planetary bodies and their loss to space or the subsurface? Mars preserves a relatively pristine, long-lived record of surface/atmosphere interactions, representing a record of a planet that once had abundant water, a thicker atmosphere, and other variables that generated habitats that could have allowed prebiotic chemistry. Why and how Mars sustained such conditions is one of the great unsolved questions of decades of exploration but there are new clues based on the role of clouds, reduced gases, and episodic volcanic and impact forcings, revealed by the geologic and isotopic record (Wordsworth, 2016; Wordsworth et al., 2017; Tosca et al., 2018). 
Venus, Earth and Mars likely began with similar climates, but evolved along very different paths, highlighting how sensitive climate may be to different forces. For example, portions of the ancient Mars surface indicate the early presence of a strong global magnetic field. Was its demise the opening to the loss of Mars' atmosphere? Building an understanding of how the planets evolved differently from each other informs our understanding of how terrestrial planets, including Earth, evolve and adapt through changes forced on them. Additionally, studying exchanges between the surface and the atmosphere is crucial to detailing the variables needed for habitability. This study is complicated on Earth because processes are masked by the presence of liquid water and surface life. By contrast, surface/atmosphere interactions can be observed directly on Mars as gases are adsorbed onto surface grains or in subsurface reservoirs. Key physical attributes that contribute to moving water back and forth (e.g., surface composition, texture/graininess of surface material, surface temperature) can be measured on modern Mars, and once the driving factors are established, processes can be extrapolated back into the past.

Late Amazonian Climate ( $\leq \mathbf{1 0 0 s}$ of Ma). What is the evidence and what are the drivers for geologically recent climate change? What characterizes the climate of icy worlds? As a terrestrial planet that - like Earth - has obliquity-driven climate cycles, Mars illuminates both questions. Mars has a record of ice age cycles; the polar layered deposits (PLD) in particular are thought to be a very direct record of climate cycles, but the landforms and subsurface ice deposits in the midlatitudes also record such events. If the PLD can be considered analogous to terrestrial ice fields, then Mars represents only the second accessible and readable planetary climate record that stretches over orbital parameter cycles that can be tied to atmospheric dynamics.

Modern Mars Climate: Mars represents a unique place to test atmospheric dynamics models. Mars is active today: sand dunes move, subliming $\mathrm{CO}_{2}$ fractures the springtime ice cover, rocks and ice blocks fall. Dust storms, sometimes of planetary scale, come and go. All of this is amenable to modeling with our tools and yet different enough that such phenomena truly test our tools, a crucial requirement if we are to extrapolate with confidence back into the past.

Modern Mars is also a testing ground for processes that are not Earthlike. On Mars, a large fraction of the main atmospheric constituent, $\mathrm{CO}_{2}$, freezes out during cold seasons, analogous to processes on bodies such as Europa, Triton and Pluto, with their even thinner atmospheres. Also, a wide range of current features on Mars, including gullies, recurring slope lineae, and erosional landforms due to $\mathrm{CO}_{2}$ frost, appear similar to Earth phenomena, but may result from different processes, thus providing a wider planetary analogy. For example, subliming frost has been shown to induce large-scale avalanches with sinuous channels and other "fluvial" features (Dundas et al., 2017) and allow $\mathrm{CO}_{2}$ ice blocks to "hovercraft" down slopes (Diniega et al., 2013). Such sublimation-driven processes are analogs to planets with migrating seasonal frost, like Pluto.

\section{Mars is a distinctive and key data point for exploring the internal structure, origin and evolution of terrestrial planets.}

What was the timing of early solar system processes, including origin and dynamic evolution? How do planets lose their heat? How old are planetary surfaces? What was the early inventory of water in planetary interiors in the inner solar system? How and when do planetary bodies accrete volatile species? Mars is a model for planetary processes and evolution (e.g. a planet with an atmosphere, magnetic field, and evolving climate) that nevertheless retains most of its geologic record, making it a testing ground for understanding geology on other planets besides Earth.

A fundamental goal of planetary science is understanding how planets form, differentiate and evolve. Clues to these processes can be drawn from understanding the history and present form of 
a planet's bulk composition, structure and thermodynamic state, but poor constraints on primary processes and the conditions under which they operated, hampers interpretations of the observed variations among the four terrestrial planets. Relative to Venus and Mercury, Mars is accessible and hospitable for robotic, and potentially crewed, surface missions. Its level of historical thermal and geological activity, intermediate between the Earth and the Moon, is ideal in attempting both to elucidate the initial conditions and to understand the subsequent processes that have led to the solar system we inhabit today. The study of isotopic and rare element systems in samples returned from Mars will make a huge contribution to understanding this history, but to understand Mars as a system requires a systematic approach to Mars' geology.

The diversity of Mars' geologic record is rivalled only by Earth; its archive includes a record of nearly every process seen on the terrestrial planets, from fluvial systems spanning $100 \mathrm{~s} \mathrm{of} \mathrm{km}$, to a wide range of volcanic emplacement styles, to migrating dunes, to massive impact basins. Geology provides answers to questions of where water existed on Mars at various times and how it cycled, as Mars is the only terrestrial planet we know of with an ancient and modern record of sediments (Groztinger \& Milliken, 2012). Volcanism, impact bombardment, fluvial and groundwater activity, and water/atmosphere interaction are recorded in the rock record, including the feedbacks with internal evolution that may have allowed and sustained habitability. These rocks are time-correlative with the Hadean/early Archean, meaning (a) the period of time when life arose on Earth is recorded on Mars; (b) if life arose on Mars at that time, then the record may still be there; (c) the influence of accretion, impacts, volcanism, and atmospheric loss drivers on the climate system can be traced in the isotopic record, just as it is on Earth.

\section{Mars provides a laboratory for characterizing how the solar system is representative of planetary systems in general, specifically in terms of habitable zones.}

How can the search for life in the solar system inform the search for habitability and evidence of life elsewhere in the universe? What are the implications of other planetary system geometries for the origin and evolution of our own solar system and vice versa? Our search for Earth-like exoplanets must include a deep understanding of what did or did not happen at Mars, and when, that ultimately yielded such a different outcome from Earth.

The search for exoplanets has revealed thousands of worlds in a wide variety of star systems; many of these planets might lie within a "habitable zone" (the zone around a star within which a planet with sufficient atmospheric pressure could potentially support liquid water on the surface). However, our understanding of what constitutes a habitable zone is still nascent. A fundamental issue is constraining the variables crucial to habitability that are solar system-scale (e.g., position with respect to the Sun, position with respect to other bodies or potential projectiles; mass and composition). For example, is small mass fatal? Mass is related to the presence and nature of a magnetic field, retention of an atmosphere, which atmospheric components are retained, and how long a body remains geologically active (thus potentially driving processes such as volcanism and plate tectonics that influence volatile and rock recycling). For example, if Mars had the same mass as Earth, even with its different position with respect to the Sun, would it be habitable today in a way comparable to Earth? The answer has key implications for the search for habitable exoplanets because it defines the boundaries and timeline of a habitable zone, as well as the constraints of mass on habitable planets. Consequently, understanding the mechanisms that drive the differences between Earth and Mars evolution is crucial to understanding whether Earth is distinctive in its relatively long-term habitability. We observe only snapshots of random environmental states from exoplanets, but Mars retains a long record of its geologic history as a habitable environment. 


\section{Mars is a long-term strategic goal for human exploration.}

Mars is the closest planet that is potentially habitable by humans in the future. What knowledge is needed to design and implement human missions to the vicinity or surface of Mars, or to design and implement sustained human presence on that body with acceptable cost, risk, and performance? How would humans change planetary environments over time? Mars is the horizon goal of human exploration, as recognized by NASA's Science Vision 2020-2024 (https://science.nasa.gov/aboutus/science-strategy). As such, we must not only understand how to get there, but we must identify the high-priority science that is best facilitated by in situ exploration of Mars and its environs specifically by humans. As the enabling technology that will carry humans to Mars is rapidly evolving, it is crucial to move forward now to define the precursor research and missions needed to acquire information concerning potential resources and map out the science to be executed; to perform technology and flight system demonstrations; and to deploy infrastructure to support future human exploration activities. We must understand as much as we can about the planet before a human presence there begins to alter it, even as we plan the science that may only be possible when there are humans on Mars to carry it out.

\section{Conclusions}

Mars science is at a turning point, similar to that seen in terrestrial geoscience in the $1800 \mathrm{~s}$. At that time, Earth geologic research was primarily descriptive; scientists had increasingly refined observations, but not an understanding of the processes that drove them. Now, for Mars, we are in a similar place; we are able to recognize evidence in Mars' geologic record of potentially habitable environments, of a changing, evolving climate, and of ancient archives of planetary origin and evolution. Furthermore, we can directly observe present-day planetary processes (some with terrestrial analogs, and some with analogs to other solar system bodies) and couple such observations to measurements of environmental conditions. The next step is to tie observation and process to interpretation. Samples carefully selected by the Perseverance rover and returned to Earth for sophisticated laboratory analyses (cf. McSween et al., 2020) will usher in a new age of Mars science akin to the advances in lunar science made with Apollo samples over the past half century. But exploration does not and cannot end there as the record of driving processes requires tracing the planet's history over time and space as different pieces of the Martian record provide different clues to its evolution. As on Earth, where we have built up an understanding of the framework for life and habitability through multiple observations over diverse locations through a wide range of techniques, we are now on the cusp of exploring Mars as a system - not just by discrete discipline, but as a planetary body of complex, interconnected processes. This is the understanding required for us to continue targeting Mars as an objective for astrobiology and a compelling witness plate for planetary evolution.

References: Hubbard, S. (2011), Exploring Mars: Chronicles from a Decade of Discovery, University of Arizona Press.

Additional references compiled at: https://mepag.jpl.nasa.gov/reports/decadal20232032/MarsCompellingTargetReferences.pdf. 